Pacific Journal of Mathematics

SEQUEL TO A PAPER OF A. E. TAYLOR 


\section{SEQUEL TO A PAPER OF A. E. TAYLOR}

\section{EARL BERKSON}

Introduction. In $\S 7$ of the paper of the title [3], certain questions are posed. The theorem presented in $\S 1$ of this sequel answers these questions. The presentation in $\S 1$ proceeds independently of [3] and is self-contained.

The notions of Mittag-Leffler development and finite type, as introduced in [3], call for some comment; in fact the notion of finite type is not made entirely clear in [3]. These and related matters are taken up in $\S \S 2$ and 3 below.

In this sequel to Taylor's paper, all convergence of operators is with respect to the uniform operator topology.

1. Theorem. Let $X$ be a complex Banach space, and $E_{1}, E_{2}, \ldots$ an infinite sequence of bounded non-zero projections of $X$ into itself. Suppose $\left\{\lambda_{n}\right\}$ is a sequence of complex numbers such that the series

$$
\sum_{n=1}^{\infty} \lambda_{n} E_{n}
$$

converges to an operator $B$. Then

$$
\lambda_{n} \rightarrow 0 \text {. }
$$

If, in addition to the above hypotheses, $E_{m} E_{n}=0$ for $m \neq n$, and the $\lambda_{n}$ 's are distinct and non-zero, then:

(1.3) The spectrum of $B, \sigma(B)$, is the set of points $\left\{0, \lambda_{1}, \cdots, \lambda_{n}, \cdots\right\}$. In view of (1.2), 0 is the sole accumulation point of $\sigma(B)$.

(1.4) The resolvent of $B, R_{\lambda}(B)$, is given by

$$
R_{\lambda}(B)=\frac{I}{\lambda}+\sum_{n=1}^{\infty} \frac{\lambda_{n}}{\lambda\left(\lambda-\lambda_{n}\right)} E_{n},
$$

where $I$ is the identity operator and the series converges uniformly with respect to $\lambda$ on each compact subset of the resolvent set, $\rho(B)$.

(1.5) Each of the points $\lambda_{n}$ is a simple pole of $R_{\lambda}(B)$, and the residue of $R_{\lambda}(B)$ at $\lambda_{n}$ is $E_{n}$.

Proof. For each $n$ the idempotence of $E_{n}$ implies that $\left\|E_{n}\right\| \leq$ $\left\|E_{n}\right\|^{2}$, whence, since $E_{n} \neq 0,\left\|E_{n}\right\| \geq 1$. Hence

Received January 12, 1960. 


$$
\left\|\lambda_{n} E_{n}\right\| \geq\left|\lambda_{n}\right| \text { for each } n \text {. }
$$

Since (1.1) converges, the sequence of its terms, $\left\{\lambda_{n} E_{n}\right\}$, tends to 0 , and so by (1.6), $\lambda_{n} \rightarrow 0$. This completes the demonstration of (1.2).

Henceforth in this proof we assume the additional hypotheses that $E_{m} E_{n}=0$ for $m \neq n$, and that the $\lambda_{n}$ 's are distinct and non-zero. We introduce some notation. For each positive integer $k$, let

$$
\begin{aligned}
B_{k} & =\sum_{n=1}^{k} \lambda_{n} E_{n} ; \\
T_{k}(\lambda) & =\frac{I}{\lambda}+\sum_{n=1}^{k} \frac{\lambda_{n}}{\lambda\left(\lambda-\lambda_{n}\right)} E_{n}, \text { for } \lambda \neq 0, \lambda_{1}, \cdots, \lambda_{k} .
\end{aligned}
$$

Obviously each $\lambda_{n}(1 \leq n \leq k)$ is an eigenvalue of $B_{k}$. Since the range of $E_{m}$ for $m>k$ is contained in the null space of $B_{k}, 0$ is in $\sigma\left(B_{k}\right)$. Thus,

$$
\left\{0, \lambda_{1}, \cdots, \lambda_{k}\right\} \subset \sigma\left(B_{k}\right) .
$$

One sees directly that if $\lambda \neq 0, \lambda_{1}, \cdots, \lambda_{k}$,

$$
\begin{aligned}
T_{k}(\lambda)\left(\lambda I-B_{k}\right) & =\left(\lambda I-B_{k}\right) T_{k}(\lambda) \\
& =I-\sum_{n=1}^{k} \frac{\lambda_{n}}{\lambda} E_{n}+\sum_{n=1}^{k} \frac{\lambda_{n}}{\lambda-\lambda_{n}} E_{n}-\sum_{n=1}^{k} \frac{\lambda_{n}^{2}}{\lambda\left(\lambda-\lambda_{n}\right)} E_{n} .
\end{aligned}
$$

But for each $n$,

$$
-\frac{\lambda_{n}}{\lambda}+\frac{\lambda_{n}}{\lambda-\lambda_{n}}-\frac{\lambda_{n}^{2}}{\lambda\left(\lambda-\lambda_{n}\right)}=0
$$

Hence

$$
T_{k}(\lambda)\left(\lambda I-B_{k}\right)=\left(\lambda I-B_{k}\right) T_{k}(\lambda)=I, \text { for } \lambda \neq 0, \lambda_{1}, \cdots, \lambda_{k} .
$$

We can summarize (1.7) and (1.8) by

$$
\text { For each } k, \sigma\left(B_{k}\right)=\left\{0, \lambda_{1}, \cdots, \lambda_{k}\right\} \text { and } T_{k}(\lambda)=R_{\lambda}\left(B_{k}\right) \text {. }
$$

We now make use of the following theorem due to J. D. Newburgh [1]: If, in a Banach algebra, $\left\{x_{k}\right\}$ is a sequence convergent to an element $x$ of the algebra, and, for all $k, x_{k} x=x x_{k}$, then $\sigma\left(x_{k}\right) \rightarrow \sigma(x)$ in the Hausdorff metric for compact subsets of the complex plane.

As a result of this theorem and (1.9),

$$
\sigma(B)=\lim _{k} \sigma\left(B_{k}\right)=\lim \left\{0, \lambda_{1}, \cdots, \lambda_{k}\right\}=\left\{0, \lambda_{1}, \cdots, \lambda_{n}, \cdots\right\} .
$$

This settles (1.3).

For any $\lambda$ in $\rho(B)$ : 


$$
\begin{aligned}
T_{k}(\lambda)(\lambda I-B) & =T_{k}(\lambda)\left(\lambda I-B_{k}\right)-T_{k}(\lambda) \sum_{n=k+1}^{\infty} \lambda_{n} E_{n} \\
& =I-T_{k}(\lambda) \sum_{n=k+1}^{\infty} \lambda_{n} E_{n}, \quad \text { by }(1.9) . \\
T_{k}(\lambda) \sum_{n=k+1}^{\infty} \lambda_{n} E_{n} & =\left(\frac{I}{\lambda}-\sum_{n=1}^{k} \frac{\lambda_{n}}{\lambda\left(\lambda-\lambda_{n}\right)} E_{n}\right) \sum_{n=k+1}^{\infty} \lambda_{n} E_{n} \\
& =\frac{1}{\lambda} \sum_{n=k+1}^{\infty} \lambda_{n} E_{n} .
\end{aligned}
$$

So,

$$
T_{k}(\lambda)(\lambda I-B)=I-\frac{1}{\lambda} \sum_{n=k+1}^{\infty} \lambda_{n} E_{n} .
$$

Multiplying by $R_{\lambda}(B)$ on the right and transposing,

$$
R_{\lambda}(B)-T_{k}(\lambda)=\frac{1}{\lambda}\left(\sum_{n=k+1}^{\infty} \lambda_{n} E_{n}\right) R_{\lambda}(B) .
$$

Since $1 / \lambda$ and $R_{\lambda}(B)$ are bounded on compact subsets of $\rho(B), T_{k}(\lambda) \rightarrow$ $R_{\lambda}(B)$ uniformly on compact subsets of $\rho(B)$. This proves (1.4).

For convenience (1.5) will be demonstrated for $n=1$. The details for arbitrary $n$ are entirely analogous. To begin with, by (1.3), $\lambda_{1}$ is an isolated point of $\sigma(B)$, and hence certainly is an isolated singularity of $R_{\lambda}(B)$. Let $\Omega$ be an open disk centered at $\lambda_{1}$, which does not contain 0 or any $\lambda_{n}$ for $n \geq 2$. Then throughout $\Omega$ except at $\lambda_{1}$,

$$
\begin{aligned}
R_{\lambda}(B) & =\frac{I}{\lambda}+\sum_{n=1}^{\infty} \frac{\lambda_{n}}{\lambda\left(\lambda-\lambda_{n}\right)} E_{n} \\
& =\frac{\lambda_{1}}{\lambda\left(\lambda-\lambda_{1}\right)} E_{1}+\frac{I}{\lambda}+\sum_{n=2}^{\infty} \frac{\lambda_{n}}{\lambda\left(\lambda-\lambda_{n}\right)} E_{n} \\
& =\frac{1}{\lambda-\lambda_{1}} E_{1}-\frac{1}{\lambda} E_{1}+\frac{I}{\lambda}+\sum_{n=2}^{\infty} \frac{\lambda_{n}}{\lambda\left(\lambda-\lambda_{n}\right)} E_{n} .
\end{aligned}
$$

Let

$$
B^{\prime}=\sum_{n=2}^{\infty} \lambda_{n} E_{n}
$$

Applying our previous results to $B^{\prime}$, we have:

$$
\begin{aligned}
\sigma\left(B^{\prime}\right) & =\left\{0, \lambda_{2}, \cdots, \lambda_{n}, \cdots\right\} . \\
R_{\lambda}\left(B^{\prime}\right) & =\frac{I}{\lambda}+\sum_{n=2}^{\infty} \frac{\lambda_{n}}{\lambda\left(\lambda-\lambda_{n}\right)} E_{n} .
\end{aligned}
$$

Thus the series on the right of (1.10) converges for $\lambda=\lambda_{1}$, and the right-hand side of (1.10) with the term $\left\{1 /\left(\lambda-\lambda_{1}\right)\right\} E_{1}$ deleted is defined throughout $\Omega$ and analytic there. It is now evident from the preceding 
sentence and (1.10) that the singular part of the Laurent development of $R_{\lambda}(B)$ centered at $\lambda_{1}$ consists of the single term $\left\{1 /\left(\lambda-\lambda_{1}\right)\right\} E_{1}$. This settles (1.5) and completes the proof of the theorem.

2. In this section, free use is made of the notation and terminology of [3], and explanations of these, except in special instances, are not repeated. Specific references to [3] are italicized (e.g., "(6.2)" refers to equation 2 of $\S 6$ in [3]); references to parts of this sequel are not italicized.

Let $A$ be as described in the first paragraph of [3]. A MittagLeffler development of $R_{\lambda}(A)$ is defined in [3] (and we shall follow this definition throughout this paper) as a representation of the form (1.2) or (2.3), wherein the series involving the $E_{n}$ 's converges uniformly on each compact subset of $\rho(A)$. We shall refer to this series associated with the development. A Mittag-Leffler development of the form (1.16) is defined as a development of finite order $p$. It is important to note that the definition of Mittag-Leffler development does not require that the associated series converge absolutely, or that it converge in every rearrangement. Such developments will exist, as the classical proofs of Mittag-Leffler's theorem show; in fact, application of the results of these proofs yields a Mittag-Leffler development such that the associated series and all its rearrangements converge to the same function uniformly and absolutely on compact subsets of $\rho(A)$, but there is no requirement in the definition that a Mittag-Leffler development be obtained by applying the results of the classical proofs of Mittag-Leffler's theorem. Indeed, in $\S 3$ we shall present an example of a Mittag-Leffler development of order 1 whose associated series has a rearrangement which diverges at every point of the resolvent set. The fact that a Mittag-Leffler development, and, in particular, a development of finite order, can have an associated series with divergent rearrangements creates difficulties with the definition of "finite type." Even if $R_{\lambda}(A)$ has a Mittag-Leffler development of the form (1.16), it need not have a development of the same finite order with respect to a different enumeration of the nonzero points of $\sigma(A)$, for this is precisely a matter of rearranging the series in (1.16) without altering its value or affecting uniform convergence on compact subsets of $\rho(A)$. Thus the definition of the assertion, " $A$ is of finite type $p$," for $A$ as described in the first paragraph of [3], is not proper, since the defining conditions depend not only on $A$, but also on the enumeration of the non-zero points of $\sigma(A)$. In fact, the operator of the previously mentioned example will be of finite type 1 with respect to one enumeration of the non-zero points of its spectrum, but will not be of finite type 1 with respect to the enumeration corresponding to the rearranged series which diverges at every point of the resolvent set. 
These difficulties disappear as soon as one includes the dependence on enumeration in the concept of finite type. Specifically:

Let $A$ be a bounded linear operator mapping a Banach space into itself and possessing a denumerably infinite spectrum with 0 as sole point of accumulation. Further, let each non-zero point of $\sigma(A)$ be a simple pole of the resolvent. We enumerate these poles in some definite order $\lambda_{1}, \lambda_{2}, \cdots$, and we let $E_{n}$ be the residue at $\lambda_{n}$. Then $A$, relative to this enumeration, is said to be of finite type if there exists a positive integer $p$ such that

$$
R_{\lambda}(A)=\frac{I}{\lambda}+\cdots+\frac{A^{p-1}}{\lambda^{p}}+\sum_{n=1}^{\infty} \frac{\lambda_{n}^{p}}{\lambda^{p}\left(\lambda-\lambda_{n}\right)} E_{n},
$$

where the series converges uniformly on compact subsets of the resolvent set. The minimal $p$ for which this situation holds is then the type, relative to the enumeration $\lambda_{1}, \lambda_{2}, \cdots$.

It is to be noted that in $\S \S 1-5$ the author of [3] has a fixed enumeration in mind, and so no serious difficulties arise. Consider now the operator $B$ as described in the first paragraph of $\S 6$. In [3], the question is posed, "Is $B$ of finite type 1?" This question is ambiguous, as it stands. It is evident, however, from the statement of Lemma 6.1 that the intent of the question is:

Is it true that $\sigma(B)$ consists of 0 and the $\lambda_{n}$ 's and that $B$ is of finite type 1 with respect to the given enumeration $\lambda_{1}, \lambda_{2}, \cdots$ ?

The theorem in $\S 1$ of this sequel gives an affirmative answer.

3. We shall now give a concrete example of a Mittag-Leffler development whose associated series has divergent rearrangements; to be more precise, we shall obtain a Mittag-Leffler development of finite order 1 for the resolvent of a certain operator $B$, and show that the series associated with this development has a rearrangement which diverges at every point of the resolvent set of $B$.

Consider the Banach space $l^{1}$. For each positive integer $n$, define the bounded linear functional $f_{n}$ on $l^{1}$ by:

$$
\begin{aligned}
f_{n}\left(\left\{\alpha_{k}\right\}\right)=(-1)^{n} \sum_{k=1}^{n}(-1)^{k} \alpha_{k}, & \text { for each sequence of complex } \\
& \text { number }\left\{\alpha_{k}\right\} \text { belonging to } l^{1} .
\end{aligned}
$$

Also, for each positive integer $n$, let $e_{n}=\left\{\alpha_{k}^{(n)}\right\}$, where

$$
\alpha_{k}^{(n)}= \begin{cases}1, & \text { for } k=n \\ 0, & \text { for } k \neq n\end{cases}
$$

Finally, for each positive integer $n$, let $E_{n}$ be the following bounded linear operator mapping $l^{1}$ into itself: 


$$
E_{n}(x)=f_{n}(x)\left(e_{n}+e_{n+1}\right) \text {, for each vector } x \text { in } l^{1} .
$$

One verifies directly that each $E_{n}$ is idempotent and non-zero and that $E_{m} E_{n}=0$ if $m \neq n$ (consider the cases $m<n$ and $m>n$ separately). Note that

$$
E_{n}\left(e_{m}\right)=0 \text {, if } m>n .
$$

Consider now a sum of the form

$$
\lambda_{n+1} E_{n+1}+\lambda_{n+2} E_{n+2}+\cdots+\lambda_{n+p} E_{n+p},
$$

where $n, p$ are arbitrary positive integers, and the complex numbers $\lambda_{n+1}, \cdots, \lambda_{n+p}$ are arbitrary. If $1 \leq k \leq n+1$,

$$
\begin{aligned}
\left(\sum_{j=1}^{p} \lambda_{n+j} E_{n+j}\right) e_{k} & =\sum_{j=1}^{p} \lambda_{n+j} f_{n+j}\left(e_{k}\right)\left(e_{n+j}+e_{n+i+1}\right) \\
& =\sum_{j=1}^{p} \lambda_{n+j}(-1)^{n+j}(-1)^{k}\left(e_{n+j}+e_{n+j+1}\right) \\
& =(-1)^{n+k-1} \sum_{j=1}^{p}(-1)^{j+1} \lambda_{n+j}\left(e_{n+j}+e_{n+j+1}\right) .
\end{aligned}
$$

If we collect coefficients for each $e_{n+j}$ in the last sum, we get:

$$
\begin{aligned}
\left(\sum_{j=1}^{p} \lambda_{n+j} E_{n+j}\right) e_{k}=(-1)^{n+k-1}\left[\lambda_{n+1} e_{n+1}\right. & +\sum_{j=2}^{p}(-1)^{j}\left(\lambda_{n+j-1}-\lambda_{n+j}\right) e_{n+j} \\
& \left.+(-1)^{p+1} \lambda_{n+p} e_{n+p+1}\right] .
\end{aligned}
$$

Thus

$$
\begin{array}{r}
\left\|\left(\lambda_{n+1} E_{n+1}+\cdots+\lambda_{n+p} E_{n+p}\right) e_{k}\right\|=\left|\lambda_{n+1}\right|+\left|\lambda_{n+2}-\lambda_{n+1}\right| \\
+\left|\lambda_{n+3}-\lambda_{n+2}\right|+\cdots+\left|\lambda_{n+p}-\lambda_{n+p-1}\right|+\left|\lambda_{n+p}\right|, \\
\text { for } 1 \leq k \leq n+1 .
\end{array}
$$

If $n+1<k \leq n+p$, then by (3.1),

$$
\left(\lambda_{n+1} E_{n+1}+\cdots+\lambda_{n+p} E_{n+p}\right) e_{k}=\left(\lambda_{k} E_{k}+\cdots+\lambda_{n+p} E_{n+p}\right) e_{k} .
$$

If we adapt (3.2) to the operator $\lambda_{k} E_{k}+\cdots+\lambda_{n+p} E_{n+p}$ acting on $e_{k}$, we obtain an expression for $\left\|\left(\lambda_{k} E_{k}+\cdots+\lambda_{n+p} E_{n+p}\right) e_{k}\right\|$ which is easily seen to be majorized by

$$
2\left(\sup _{1 \leq j \leq p}\left|\lambda_{n+j}\right|\right)+\left|\lambda_{n+2}-\lambda_{n+1}\right|+\left|\lambda_{n+3}-\lambda_{n+2}\right|+\cdots+\left|\lambda_{n+p}-\lambda_{n+p-1}\right| .
$$

Combining this result with (3.2) we have:

$$
\begin{aligned}
& \left\|\left(\lambda_{n+1} E_{n+1}+\cdots+\lambda_{n+p} E_{n+p}\right) e_{k}\right\| \\
& \quad \leq 2\left(\sup _{1 \leq j \leq p}\left|\lambda_{n+j}\right|\right)+\sum_{j=1}^{p-1}\left|\lambda_{n+j+1}-\lambda_{n+j}\right|, \\
& \text { for } 1 \leq k \leq n+p .
\end{aligned}
$$


By (3.1), each $e_{k}$ for $k>n+p$ is in the null space of $\lambda_{n+1} E_{n+1}+\cdots+$ $\lambda_{n+p} E_{n+p}$. Certainly, then, (3.3) holds for every $e_{k}$. It is well known (see, for example, [4], §4.51) that if $T$ is a bounded linear operator mapping $l^{1}$ into itself, then

$$
\|T\|=\sup _{k}\left\|T e_{k}\right\| .
$$

Hence,

$$
\begin{aligned}
\left\|\lambda_{n+1} E_{n+1}+\cdots+\lambda_{n+p} E_{n+p}\right\| \\
\quad \leq 2\left(\sup _{1 \leq j \leq p}\left|\lambda_{n+\jmath}\right|\right)+\sum_{j=1}^{p-1}\left|\lambda_{n+j+1}-\lambda_{n+j}\right|,
\end{aligned}
$$

for arbitrary $n, p$ and complex numbers $\lambda_{n+1}, \cdots, \lambda_{n+p}$.

Also, from (3.2) we easily infer

$$
\left\|\lambda_{n+1} E_{n+1}+\cdots+\lambda_{n+p} E_{n+p}\right\| \geq \sum_{j=1}^{p-1}\left|\lambda_{n+j+1}-\lambda_{n+j}\right| .
$$

Recalling that an infinite sequence of complex numbers $\left\{\lambda_{n}\right\}$ is said to be of bounded variation if and only if

$$
\sum_{n=1}^{\infty}\left|\lambda_{n+1}-\lambda_{n}\right| \text { is convergent, }
$$

we can utilize (3.4) and (3.5) together with the first conclusion of the theorem in $\S 1$ to obtain (for the present particular $E_{n}$ 's):

(3.6) If $\left\{\lambda_{n}\right\}$ is a sequence of complex numbers, then $\sum_{n=1}^{\infty} \lambda_{n} E_{n}$ converges if and only if $\left\{\lambda_{n}\right\}$ is of bounded variation and tends to 0 .

Consider now a sum of the form

$\mu_{n_{1}} E_{n_{1}}+\mu_{n_{2}} E_{n_{2}}+\cdots+\mu_{n_{k}} E_{n_{k}}$, where $n_{1}<n_{2}<\cdots<n_{k}$ and the scalars $\mu_{n_{1}}, \cdots, \mu_{n_{k}}$ are arbitrary.

If for each $j<k$ we interpose $O E_{n_{j}+1}+\cdots+O E_{n_{j+1}-1}$ between the terms $\mu_{n_{j}} E_{n_{j}}$ and $\mu_{n_{j+1}} E_{n_{j+1}}$ and apply (3.5), we get

$$
\begin{aligned}
|| \mu_{n_{1}} E_{n_{1}}+\mu_{n_{2}} E_{n_{2}} & +\cdots+\mu_{n_{k}} E_{n_{k}}|| \\
& \geq\left|\mu_{n_{1}}\right|+2\left|\mu_{n_{2}}\right|+\cdots+2\left|\mu_{n_{k-1}}\right|+\left|\mu_{n_{k}}\right| \\
& \geq\left|\mu_{n_{1}}\right|+\left|\mu_{n_{2}}\right|+\cdots+\left|\mu_{n_{k}}\right| . \\
\text { for } n_{1} & <n_{2}<\cdots<n_{k} \text { and } \mu_{n_{1}}, \cdots, \mu_{n_{k}} \text { arbitrary. }
\end{aligned}
$$

Now let $\left\{\lambda_{n}\right\}$ be any sequence of complex numbers with the following properties:

(1) The $\lambda_{n}$ 's are distinct and non-zero.

(2) $\left\{\lambda_{n}\right\}$ is of bounded variation.

(3) $\lambda_{n} \rightarrow 0$. 
(4) $\quad \sum_{n=1}^{\infty}\left|\lambda_{2 n}\right|$ diverges.

For example, we could take the sequence $\{1 / n\}$. By (3.6), $\sum_{n=1}^{\infty} \lambda_{n} E_{n}$ converges to an operator $B$. By the theorem of $\S 1, \sigma(B)=\left\{0, \lambda_{1}, \cdots\right.$, $\left.\lambda_{n}, \cdots\right\}$ and $R_{\lambda}(B)$ has the Mittag-Leffler development of order 1:

$$
R_{\lambda}(B)=\frac{I}{\lambda}+\sum_{n=1}^{\infty} \frac{\lambda_{n}}{\lambda\left(\lambda-\lambda_{n}\right)} E_{n}
$$

We shall now show that the series in (3.8) has a rearrangement which diverges at every point in $\rho(B)$. Consider the series $\sum_{n=1}^{\infty}\left|\lambda_{2 n}\right|$. Since it is a divergent series of positive terms, there is a strictly increasing sequence of positive integers $n_{1}, n_{2}, \cdots$ such that

(1) $n_{1}=1$.

(2) $\left|\lambda_{2 n_{j}}\right|+\left|\lambda_{2 n_{j}+2}\right|+\cdots+\left|\lambda_{2 n_{j+1}-2}\right| \geq 1$, for each $j$.

For each $\lambda$ in $\rho(B)$, let $d(\lambda)=\inf _{n}\left|1 /\left(\lambda-\lambda_{n}\right)\right|$. Then for each $\lambda$ in $\rho(B)$ and for arbitrary $j$, we have by (3.7)

$$
\begin{aligned}
& \| \frac{\lambda_{2 n_{j}}}{\lambda\left(\lambda-\lambda_{2 n_{j}}\right)} E_{2 n_{j}}+\frac{\lambda_{2 n_{j}+2}}{\lambda\left(\lambda-\lambda_{2 n_{j}+2}\right)} E_{2 n_{j}+2} \\
& +\cdots \\
& \quad+\frac{\lambda_{2 n_{j+1}-2}}{\lambda\left(\lambda-\lambda_{2 n_{j+1}-2}\right)} E_{2 n_{j+1}-2} \mid \\
& \quad \geq\left|\frac{\lambda_{2 n_{j}}}{\lambda\left(\lambda-\lambda_{2 n_{j}}\right)}\right|+\left|\frac{\lambda_{2 n_{j}+2}}{\lambda\left(\lambda-\lambda_{2 n_{j}+2}\right)}\right|+\cdots+\left|\frac{\lambda_{2 n_{j+1}-2}}{\lambda\left(\lambda-\lambda_{2 n_{j+1}-2}\right)}\right| \\
& \geq \frac{d(\lambda)}{|\lambda|}\left[\left|\lambda_{2 n_{j}}\right|+\left|\lambda_{2 n_{j}+2}\right|+\cdots+\left|\lambda_{2 n_{j} 1^{-2}}\right|\right] \geq \frac{d(\lambda)}{|\lambda|}>0 .
\end{aligned}
$$

Consider now the rearrangement of the series in (3.8) obtained by writing down alternately

$$
\frac{\lambda_{2 j-1}}{\lambda\left(\lambda-\lambda_{2 j-1}\right)} E_{2 j-1}
$$

and the "block"

$$
\frac{\lambda_{2 n_{j}}}{\lambda\left(\lambda-\lambda_{2 n_{j}}\right)} E_{2 n_{j}}+\frac{\lambda_{2 n_{j}+2}}{\lambda\left(\lambda-\lambda_{2 n_{j}+2}\right)} E_{2 n_{j}+2}+\cdots+\frac{\lambda_{2 n_{j+1^{-2}}}}{\lambda\left(\lambda-\lambda_{i n_{j+1^{-2}}}\right)} E_{2 n_{j+1^{-2}}},
$$

taking $j$ successively to be 1,2 , etc. It follows from (3.9) that this rearranged series diverges for every $\lambda$ in $\rho(B)$.

Before closing, we state and prove a theorem which gives conditions on an operator $A$ sufficient to insure that any series associated with a Mittag-Leffler development of $R_{\lambda}(A)$ will have the property that it and all its rearrangements converge uniformly to the same function on compact subsets of $\rho(A)$.

TheOREM. Let $A$ be a bounded linear operator mapping a complex 
Banach space $X$ into itself, and let $A$ satisfy the following conditions:

(1) $\sigma(A)$ is denumerably infinite with 0 as sole point of accumulation.

(2) Each non-zero point of $\sigma(A)$ is a simple pole of $R_{\lambda}(A)$.

(3) There is a constant $M$ such that if $S$ is any finite set of non-zero points of $\sigma(A)$, and if $E_{\beta}$ is the residue of $R_{\lambda}(A)$ at $\beta$, then

$$
\left\|\sum_{\beta \in S} \alpha_{\beta} E_{\beta}\right\| \leq M \sup _{\beta \in S}\left|\alpha_{\beta}\right| \text {, for arbitrary scalars }\left\{\alpha_{\beta}\right\}_{\beta \in S} .
$$

Under these hypotheses, if an enumeration $\lambda_{1}, \lambda_{2}, \cdots$ of the non-zero points of $\sigma(A)$ is given, and the residue of $R_{\lambda}(A)$ at $\lambda_{n}$ is denoted by $E_{n}$, and if $R_{\lambda}(A)$ then has the Mittag-Leffler development

$$
R_{\lambda}(A)=\sum_{j=1}^{\infty} \frac{\lambda_{j}^{n_{j}}}{\lambda^{n_{j}}\left(\lambda-\lambda_{j}\right)} E_{j}+\Phi(\lambda),
$$

then we can conclude that every rearrangement of the series in (3.10) converges uniformly to $R_{\lambda}(A)-\Phi(\lambda)$ on compact subsets of $\rho(A)$.

Proof. We state first a theorem of Orlicz [2] which will be needed: If a series of vectors in a Banach space has the property that it and all its rearrangements are convergent, then it and all its rearrangements have the same sum.

Now let $K$ be a compact subset of $\rho(A)$. Since the series in (3.10) converges uniformly on $K$, we have

$$
\left\|\frac{\lambda_{j}^{n_{j}}}{\lambda^{n_{j}}\left(\lambda-\lambda_{j}\right)} E_{j}\right\| \rightarrow 0 \text { as } j \rightarrow \infty \text {, uniformly on } K \text {. }
$$

Since $\left\|E_{j}\right\| \geq 1$,

$$
\left|\frac{\lambda_{j}^{n_{j}}}{\lambda^{n_{j}}\left(\lambda-\lambda_{j}\right)}\right| \rightarrow 0 \text { uniformly on } K
$$

It is easily seen that any rearrangement of the sequence

$$
\left\{\frac{\lambda_{j}^{n_{j}}}{\lambda^{n_{j}}\left(\lambda-\lambda_{j}\right)}\right\}
$$

tends uniformly to 0 on $K$. From this remark and from Condition (3) of the hypotheses, it is easily seen that the sequence of partial sum of any rearrangement of the series in (3.10) is uniformly Cauchy on $K$. Hence every rearrangement of the series in (3.10) converges uniformly on $K$. We now need only show that every rearrangent converges to $R_{\lambda}(B)-\Phi(\lambda)$ pointwise on $K$. As is easily seen, it suffices to show that if $\mu$ is an arbitrary but fixed point of $K$, then every rearrangement of 


$$
\sum_{j=1}^{\infty} \frac{\lambda_{j}^{n_{j}}}{\mu^{n_{j}}\left(\mu-\lambda_{j}\right)} E_{j}
$$

converges to $R_{\mu}(A)-\Phi(\mu)$. By what has already been shown, we know that (3.11) and all its rearrangements converge. By the theorem of Orlicz, all the rearrangements of (3.11) converge to the value of (3.11), which, by (3.10), is precisely $R_{\mu}(A)-\Phi(\mu)$. This completes the proof of the theorem.

We remark, in closing, that if $X$ is an infinite dimensional Hilbert space, if $A$ satisfies conditions (1) and (2), and if the residue of $R_{\lambda}(A)$ at each non-zero point of $\sigma(A)$ is self-adjoint, then the hypotheses of the theorem are fulfilled.

\section{REFERENCES}

1. J. D. Newburgh, The variation of spectra, Duke Math. J., 18 (1951), 165-176.

2. W. Orlicz, Beiträge zur Theorie der Orthogonalentwicklungen II, Studia Math., 1 (1929), 241-255.

3. A. E. Taylor, Mittag-Leffler expansions and spectral theory, Pacific J. Math., 10 (1960) 000-000.

4. - Introduction to Functional Analysis, John Wiley, New York, 1958.

The University of Chicago 


\section{PACIFIC JOURNAL OF MATHEMATICS}

\section{EDITORS}

\author{
David Gilbarg \\ Stanford University \\ Stanford, California \\ F. H. Brownell \\ University of Washington \\ Seattle 5 , Washington
}

\author{
A. L. Whiteman \\ University of Southern California \\ Los Angeles 7, California \\ L. J. PAIGE \\ University of California \\ Los Angeles 24, California
}

\section{ASSOCIATE EDITORS}

\author{
E. F. BECKENBACH \\ T. M. CHERRY \\ D. DERRY
}
E. HEWITT
A. HORN
L. NACHBIN
M. OHTSUKA
H. L. ROYDEN
M. M. SCHIFFER

E. SPANIER

E. G. STRAUS

F. WOLF

\section{SUPPORTING INSTITUTIONS}

\author{
UNIVERSITY OF BRITISH COLUMBIA \\ CALIFORNIA INSTITUTE OF TECHNOLOGY \\ UNIVERSITY OF CALIFORNIA \\ MONTANA STATE UNIVERSITY \\ UNIVERSITY OF NEVADA \\ NEW MEXICO STATE UNIVERSITY \\ OREGON STATE COLLEGE \\ UNIVERSITY OF OREGON \\ OSAKA UNIVERSITY \\ UNIVERSITY OF SOUTHERN CALIFORNIA
}

\author{
STANFORD UNIVERSITY \\ UNIVERSITY OF TOKYO \\ UNIVERSITY OF UTAH \\ WASHINGTON STATE COLLEGE \\ UNIVERSITY OF WASHINGTON

AMERICAN MATHEMATICAL SOCIETY
CALIFORNIA RESEARCH CORPORATION
HUGHES AIRCRAFT COMPANY
SPACE TECHNOLOGY LABORATORIES
NAVAL ORDNANCE TEST STATION

Mathematical papers intended for publication in the Pacific Journal of Mathematics should be typewritten (double spaced), and the author should keep a complete copy. Manuscripts may be sent to any one of the four editors. All other communications to the editors should be addressed to the managing editor, L. J. Paige at the University of California, Los Angeles 24, California. ..

50 reprints per author of each article are furnished free of charge; additional copies may be obtained at cost in multiples of 50 .

The Pacific Journal of Mathematics is published quarterly, in March, June, September, and December. The price per volume (4 numbers) is $\$ 12.00$; single issues, $\$ 3.50$. Back numbers are available. Special price to individual faculty members of supporting institutions and to individual members of the American Mathematical Society: $\$ 4.00$ per volume; single issues, $\$ 1.25$.

Subscriptions, orders for back numbers, and changes of address should be sent to Pacific Journal of Mathematics, 2120 Oxford Street, Berkeley 4, California.

Printed at Kokusai Bunken Insatsusha (International Academic Printing Co., Ltd.), No. $6_{4}$ 2-chome, Fujimi-cho, Chiyoda-ku, Tokyo, Japan.

PUBLISHED BY PACIFIC JOURNAL OF MATHEMATICS, A NON-PROFIT CORPORATION

The Supporting Institutions listed above contribute to the cost of publication of this Journăl, but they are not owners or publishers and have no responsibility for its content or policies. 


\section{Pacific Journal of Mathematics}

\section{Vol. 10, No. $3 \quad$ November, 1960}

Glen Earl Baxter, An analytic problem whose solution follows from a simple

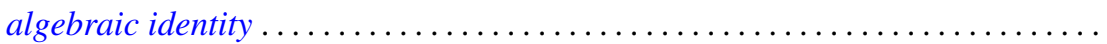

Leonard D. Berkovitz and Melvin Dresher, A multimove infinite game with linear payoff. .

Earl Robert Berkson, Sequel to a paper of A. E. Taylor ......................

Gerald Berman and Robert Jerome Silverman, Embedding of algebraic systems.... 767

Peter Crawley, Lattices whose congruences form a boolean algebra . . . . . ...... 777

Robert E. Edwards, Integral bases in inductive limit spaces . . . . . . . . . . . . . . .

Daniel T. Finkbeiner, II, Irreducible congruence relations on lattices . . . . . . . . . .

William James Firey, Isoperimetric ratios of Reuleaux polygons . . . . . . . . . . . 787

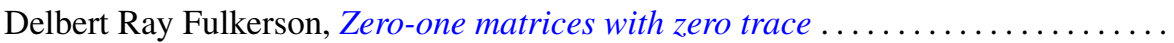

Leon W. Green, A sphere characterization related to Blaschke's conjecture........

Israel (Yitzchak) Nathan Herstein and Erwin Kleinfeld, Lie mappings in

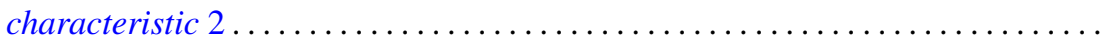

Charles Ray Hobby, A characteristic subgroup of a $p$-group .................

R. K. Juberg, On the Dirichlet problem for certain higher order parabolic

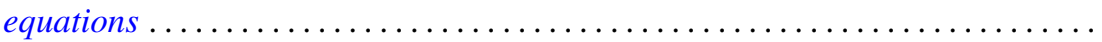

Melvin Katz, Infinitely repeatable games ......................

Emma Lehmer, On Jacobi functions . . . . . . . . . . . . . . . . . . . . . . . . .

D. H. Lehmer, Power character matrices

Henry B. Mann, A refinement of the fundamental theorem on the density of the sum of two sets of integers.

Marvin David Marcus and Roy Westwick, Linear maps on skew symmetric matrices: the invariance of elementary symmetric functions . .

Richard Dean Mayer and Richard Scott Pierce, Boolean algebras with ordered

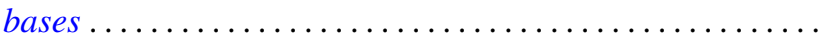

Trevor James McMinn, On the line segments of a convex surface in $E_{3} \ldots$

Frank Albert Raymond, The end point compactification of manifolds ..

Edgar Reich and S. E. Warschawski, On canonical conformal maps of regions of arbitrary connectivity

Marvin Rosenblum, The absolute continuity of Toeplitz's matrices...

Lee Albert Rubel, Maximal means and Tauberian theorems . .

Helmut Heinrich Schaefer, Some spectral properties of positive linear operators

Jeremiah Milton Stark, Minimum problems in the theory of pseudo-conformal transformations and their application to estimation of the curvature of the invariant metric.

Robert Steinberg, The simplicity of certain groups ...

Hisahiro Tamano, On paracompactness. .

Angus E. Taylor, Mittag-Leffler expansions and spectral theory .

Marion Franklin Tinsley, Permanents of cyclic matrices ...... . 